\title{
NUEVO REGISTRO DE ARTE RUPESTRE EN PASOS CORDILLERANOS: PAREDÓN LUISA (COCHAMÓ, REGIÓN DE LOS LAGOS, CHILE)
}

FRANCISCA MOYAa ${ }^{a}$ SIMÓN SIERRALTA ${ }^{b} \&$ RENATA GUTIÉRREZ

\begin{abstract}
RESUMEN
La presente nota tiene como objetivo informar respecto a un nuevo registro de arte rupestre en las cercanías del lago Vidal Gormaz, comuna de Cochamó, región de Los Lagos, Chile. El sitio Paredón Luisa presenta un paredón rocoso que conforma un alero donde se identificaron pinturas abstractas de color rojo. Tras comparar los atributos iconográficos y espaciales del sitio con las evidencias arqueológicas de la región, se propone una cronología tardía y una relación con otros contextos de bosque cordillerano ya reportados en la zona.
\end{abstract}

PALABRAS CLAVE: arte rupestre, pasos cordilleranos, cazadores-recolectores.

\section{NEW RECORD OF ROCK ART IN MOUNTAIN PASSES: PAREDÓN LUISA (COCHAMÓ, REGIÓN DE LOS LAGOS, CHILE)}

\section{ABSTRACT}

The aim of this note is to report about a new record of rock art, near Lago Vidal Gormaz, municipality of Cochamó, Los Lagos Region, Chile. The Paredón Luisa site presents a rock shelter where red abstract paintings were identified. After comparing iconographic and spatial attributes of the site with the archaeological evidence of the region, we propose a late chronology and a relationship with other contexts from the mountain forests that have already been reported in the zone.

KEY WORDS: rock art, mountain passes, hunter-gatherers.

a SERP/Secció de Prehistòria i Arqueologia, Facultat de Geografia i Història, Universitat de Barcelona. $\measuredangle$ franmoya.c@gmail.com

b Dirección Museológica, Universidad Austral de Chile, simon.sierralta@uach.cl

c Investigadora independiente, renatags@gmail.com 


\section{INTRODUCCIÓN}

En 2012 se ejecutó un proyecto de la Fundación Sendero de Chile para el acondicionamiento de un sendero pedestre binacional que recuperara para el uso turístico el camino de Cochamó, una ruta cordillerana que funcionó para el traslado de ganado desde las pampas argentinas a los embarcaderos del Pacífico hacia finales del siglo XIX (Infante, 2013). En ese marco, con el objetivo de integrarlos al guión interpretativo de la ruta, fueron visitados y registrados dos sitios con arte rupestre previamente reportados durante un Estudio de Impacto Ambiental: Torrentoso 01 y Correntoso 01 (Fig. 1) (Labarca, 2007; Bellelli et al. 2008; Podestá et al. 2008). Además, gracias a la información y guía de habitantes locales se registró un nuevo sitio con arte rupestre,
Paredón Luisa, previamente no reportado (Fig. 1). Aquí presentamos una caracterización inicial de este último sitio, con el objetivo de situarlo en relación a las evidencias previamente registradas en ambos lados de la cordillera.

El camino de Cochamó funcionó en las últimas décadas del siglo XIX y las primeras del XX, a partir de la habilitación del paso cordillerano "El León" (4130' S). Éste se localiza en la confluencia de los ríos Leones y Manso, en el sector andino de la comuna de Cochamó, provincia de Llanquihue. La ruta asciende desde las pampas orientales siguiendo el curso del río Manso inferior, que se origina en el lago Steffen $50 \mathrm{~km}$ al sur de San Carlos de Bariloche, y recorre de este a oeste un valle de unos $2 \mathrm{~km}$ de ancho hasta llegar al paso. Diez kilómetros más abajo, el Manso vira hacia el sur para desembocar en el lago Puelo y eventualmente en el océano

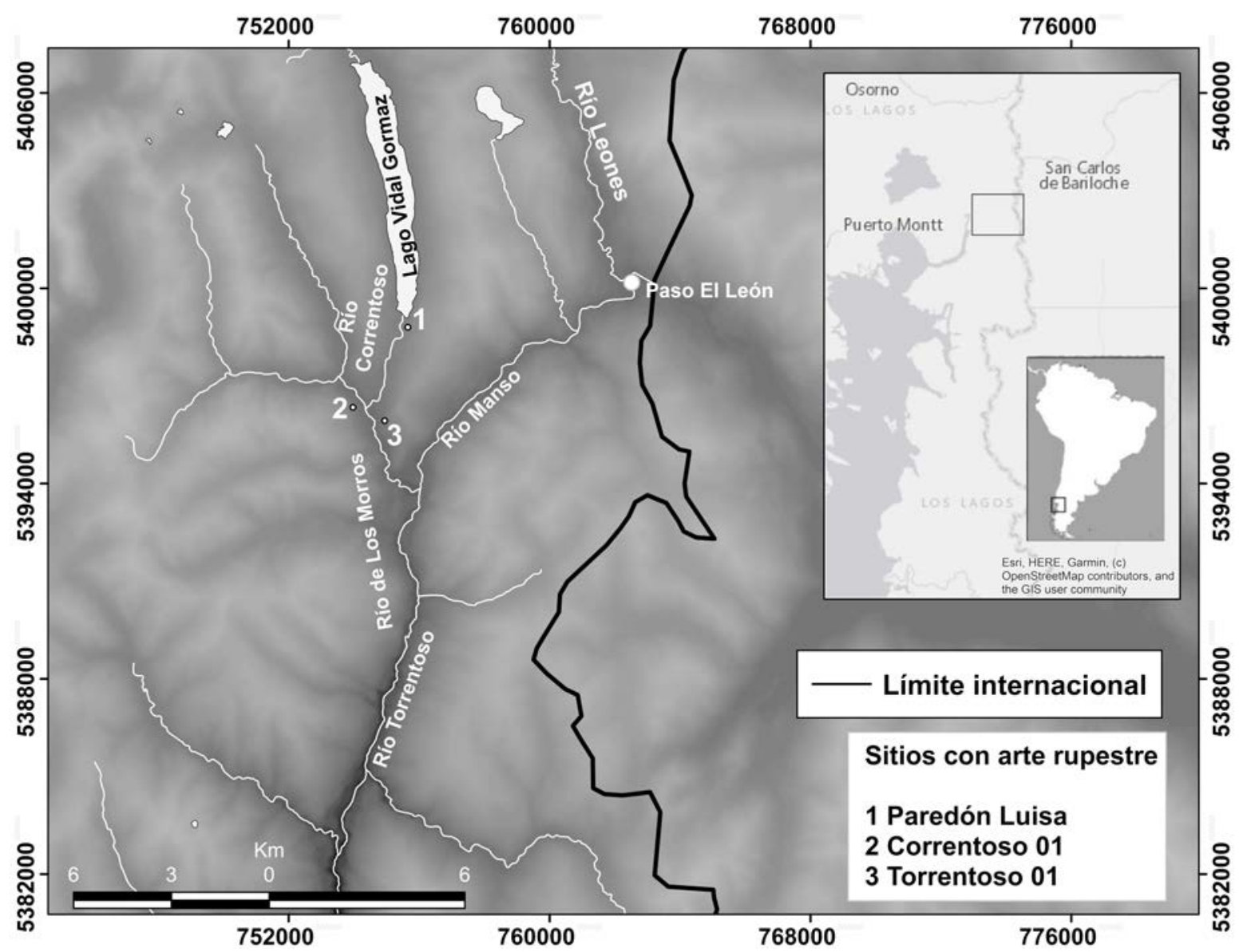

Fig. 1. Detalle de la ubicación de los sitios Paredón Luisa, Correntoso 01 y Torrentoso 01. 


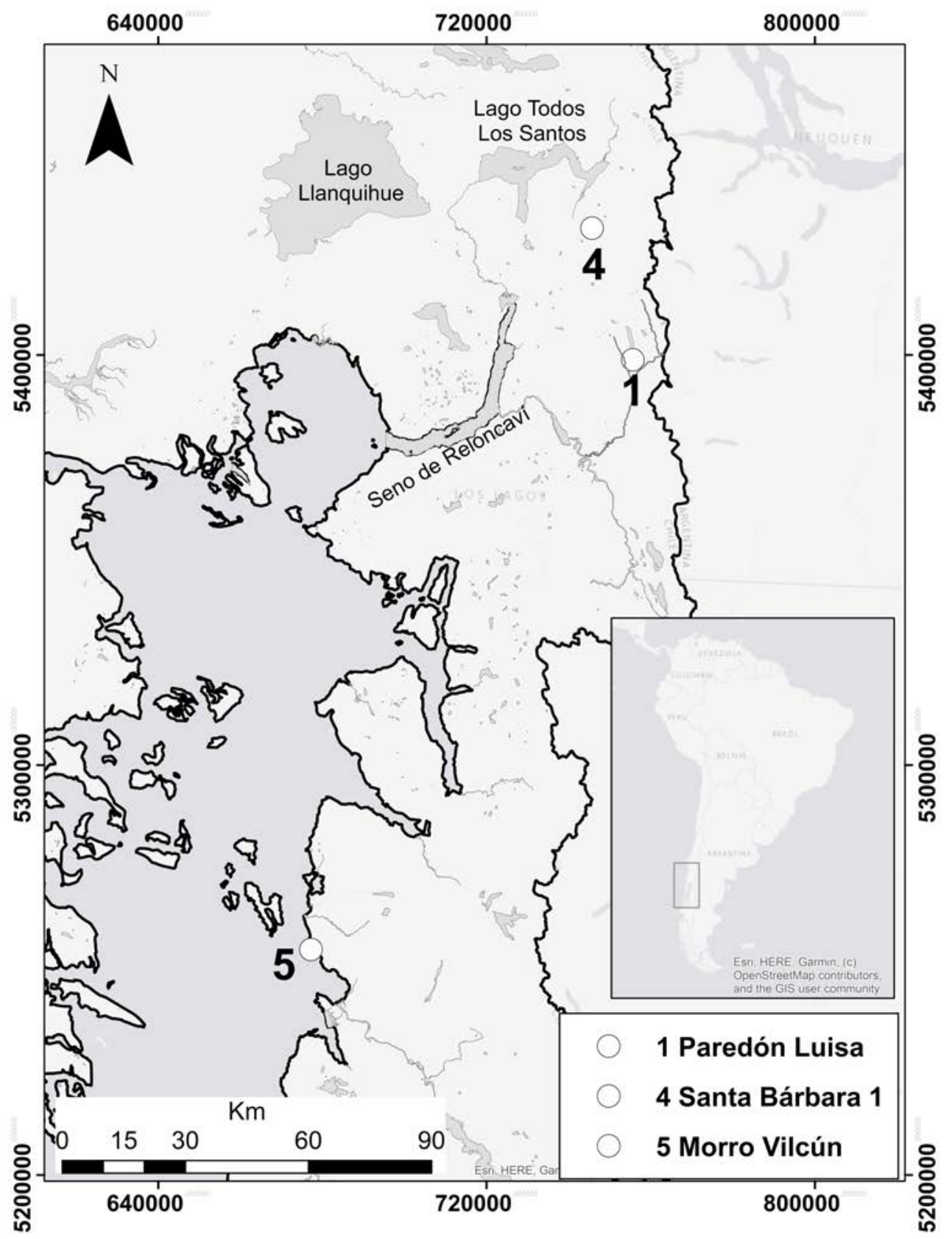

Fig. 2. Ubicación de los sitios arqueológicos que presentan arte rupestre y son mencionados en este trabajo.

Pacífico. El antiguo camino, en cambio, tornaba al norte por el río Torrentoso, para luego remontar el río Los Morros, cruzar un portezuelo y descender hacia el estuario de Reloncaví por el Cochamó (Figs. 1 y 2). El sendero actual transita solo parcialmente esta ruta, pues se desvía en el río Correntoso hacia el lago Vidal Gormaz, y vuelve a retomar la ruta histórica en el sector conocido como El Arco.

La porción occidental de la ruta está constituida por una red de escarpados valles fluvioglaciales, con una densa cobertura de selva fría valdiviana caracterizada por comunidades arbóreas de Nothofagus sp., ulmos (Eucryphia 
cordifolia), tineos (Weinmannia trichosperma), entre otros, y un denso sotobosque de helechos y colihues (Chusquea coleu). La fauna es relativamente poco densa, destacando felinos (Puma concolor, Leopardus guigna, Leopardus colocolo), cérvidos (Hippocamelus bisulcus, Pudu puda), marsupiales (Dromiciops giroides, Rhyncholestes raphanurus), otros mamíferos menores (e.g. Conepatus humboldtii), aves y anfibios. Las precipitaciones vinculadas a los vientos del oeste alcanzan medias superiores a los $2.000 \mathrm{~mm}$ anuales, presentándose durante todo el año, aunque con variaciones estacionales que determinan una disminución de lluvias hacia el verano (Moreno, 2004).

La combinación de los relieves escarpados y la cubierta selvática construyen un muro vegetal en los límites de los senderos mantenidos por los pobladores del sector, constituyendo un escenario complejo para la identificación de sitios arqueológicos. En términos prácticos, estas condiciones geográficas limitan el desarrollo de prospecciones sistemáticas que sean efectivas, de modo que los contextos que han podido ser registrados corresponden a lugares conocidos por los habitantes de la zona. De esta manera, durante el trabajo de terreno fue posible recopilar información relativa al hallazgo de fragmentos cerámicos, soportes de molienda y pipas de piedra pulida por parte de los residentes, en las inmediaciones de las casas, y de aleros con posibles indicaciones de ocupaciones humanas en distintos puntos del sector estudiado.

\section{EL SITIO PAREDÓN LUISA}

Paredón Luisa $\left(41^{\circ} 31.337\right.$ S $71^{\circ} 56.217$ O) se ubica en una ladera de cerro, en la selva lluviosa cordillerana del estuario de Reloncaví (Fig. 2). Conforma un extenso paredón rocoso de unos $10 \mathrm{~m}$ de largo, localizado a $659 \mathrm{msnm}$ en una ladera orientada al este, a ca. $300 \mathrm{~m}$ de la ribera sur del lago Vidal Gormaz. La ladera se encuentra completamente cubierta por la vegetación, lo que genera condiciones de alta humedad, baja luz y poca visibilidad. El paredón da forma a un alero mediano (Fig. 3) cuya visera posee una serie de paneles, uno de los cuales presenta un único conjunto de arte rupestre que se orienta al noroeste. En éste se identificaron cuatro representaciones de un mismo motivo geométrico en pintura roja. Los diseños se encuentran distribuidos horizontalmente en el panel, en una extensión de $40 \mathrm{~cm}$. El largo máximo de cada diseño oscila entre 10 y $15 \mathrm{~cm}$ y el ancho entre 3 y $4 \mathrm{~cm}$, aproximadamente (Fig. 4). Tres de los diseños presentaban un buen estado de conservación, siendo distinguibles de forma clara. El estado de conservación del cuarto diseño es deficiente, siendo observable solamente tras el procesamiento de las fotografías a través del plug-in Decorrelation Stretch (DStretch) del software ImageJ (Harman, 2008; Acevedo \& Franco, 2012). Esto se debe principalmente a la acción de la humedad. No se observan más diseños en el sitio, salvo algunos dibujos subactuales que representan figuras animales y humanas esquemáticas.

Por otra parte, el piso del alero presenta un depósito de materia orgánica cuyo potencial estratigráfico no pudo estimarse, y en el que no se registró material cultural superficial. No obstante, la acumulación reciente de hojas y ramas dificultaba la observación. Cabe señalar que adyacente a la ladera donde se encuentra el sitio, hay una pequeña pampa donde actualmente viven dos familias que desarrollan actividades agroganaderas de baja escala. La señora Luisa Valderas, habitante del lugar, señaló que en dicho terreno se habían hallado fragmentos cerámicos y pipas de piedra, los cuales ya no se encontraban en su poder. La distancia entre Paredón Luisa y el lugar de dichos hallazgos no supera los $200 \mathrm{~m}$ lineales.

Paredón Luisa se localiza a $3 \mathrm{~km}$ al noreste de Correntoso 01 y Torrentoso 01, los sitios previamente registrados en un Estudio de Impacto Ambiental (Podestá et al. 2008). Las figuras del sitio corresponden a diseños abstractos, lineales, conformados a partir de ángulos rectos $\mathrm{y}$, al igual que Torrentoso 01 y Correntoso 01, se pueden clasificar en el llamado "estilo de Grecas" (Menghin, 1957) o "Tendencia Abstracta Lineal Compleja" (Gradin, 1988). Esta última tiene una extensa distribución en la Patagonia argentina, y dentro de ella se ha definido la modalidad del "Ámbito Lacustre Boscoso del Noroeste de Patagonia" que tendría 

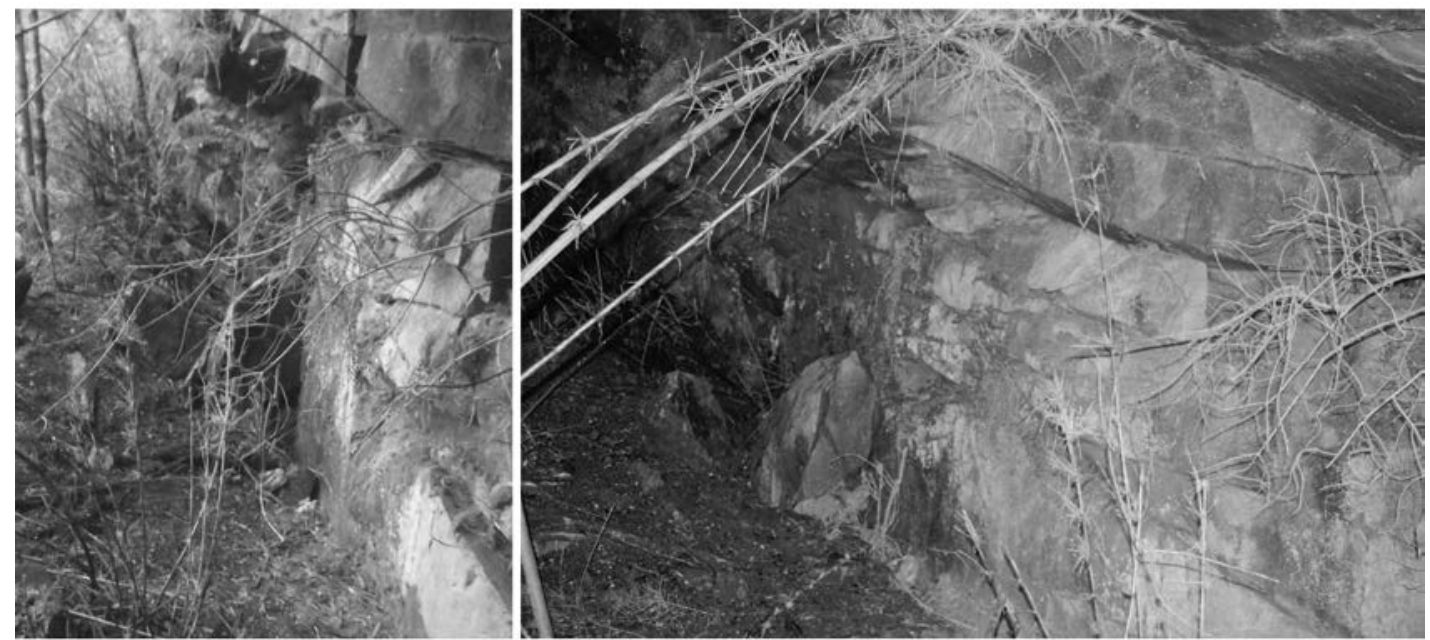

Fig. 3. Alero rocoso Paredón Luisa.
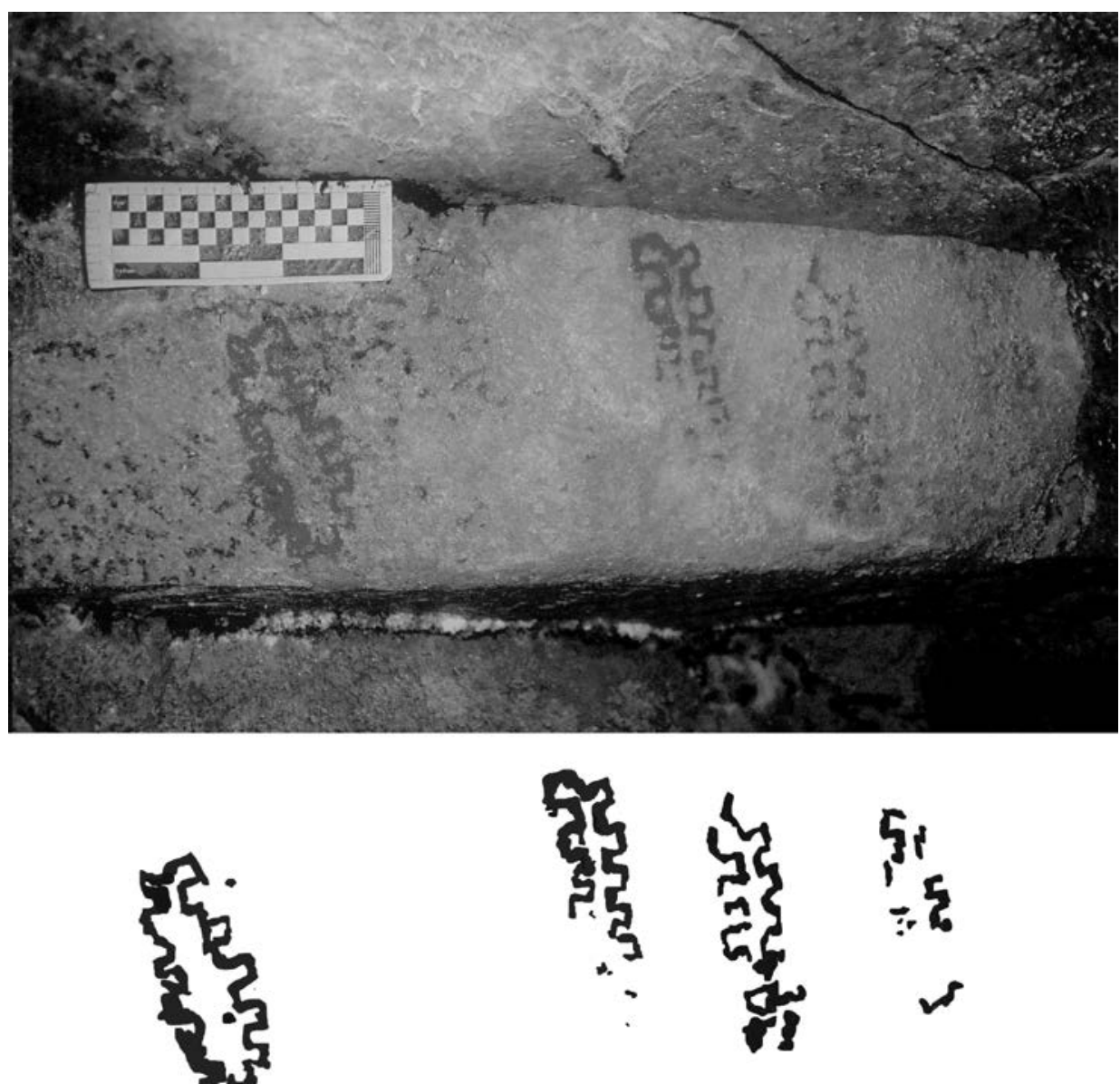

Fig. 4. Fotografía y dibujo digital del panel de arte rupestre del sitio Paredón Luisa. 
una distribución local en la cordillera de las provincias de Neuquén y Río Negro (Albornoz \& Cúneo, 2000). Paredón Luisa podría clasificarse dentro de esta modalidad, la que se caracteriza por diseños menos complejos que los de Patagonia meridional (Albornoz \& Cúneo, 2000) y que ha sido identificada en múltiples sitios del Manso Superior en la vertiente oriental de los Andes (Podestá et al. 2008).

\section{PAREDÓN LUISA}

\section{EN EL CONTEXTO REGIONAL}

El arte rupestre es una materialidad que se ha observado con baja frecuencia en la vertiente occidental de los Andes norpatagónicos. Recientemente, se han identificado pocos sitios aislados, los cuales se han comparado estilísticamente con las tradiciones definidas en las últimas décadas para la vertiente oriental de dicho macizo (Gradin, 1988; Podestá et al. 2008; Mena et al. 2011; Dillehay \& Ocampo, 2016).

En términos cronológicos, para la Tendencia Abstracta Lineal Compleja se propone una antigüedad máxima de 1.300 a 1.200 años $\mathrm{AP}$, mientras que la mayoría de los fechados de contextos estratigráficos en Patagonia septentrional (cuenca del Limay-Nahuel Huapi) rondan el lapso entre 700 y 600 años AP (Fernández et al. 2013). Bajo la premisa de que las pinturas de Paredón Luisa corresponderían a esta tendencia estilística y asumiendo la cronología que se ha propuesto para ella, se trataría de un sitio con ocupaciones al menos del Holoceno tardío.

En la misma línea, los diseños de Paredón Luisa son llamativamente similares a los del sitio Alero Rocoso Schirmer o Santa Bárbara 1, ubicado en las proximidades del lago Todos Los Santos, 35 $\mathrm{km}$ al norte y asociado también a una ruta de cruce cordillerano, en este caso el Paso Vuriloche (Fig. 2) (Dillehay \& Ocampo, 2016). Para Santa Bárbara 1 se propone un fechado prehispánico tardío o poshispánico temprano, a partir de la presencia de cerámica y los fechados de un fogón en $1.280 \pm$ 40 no cal dC (Dillehay \& Ocampo, 2016). En base a sus similitudes iconográficas y espaciales, ambos sitios podrían haber tenido una cronología similar, lo que sumado a su correspondencia con el estilo de Grecas nos lleva a proponer como hipótesis inicial que las pinturas de Paredón Luisa tendrían una data no superior al último milenio.

\section{DISCUSIÓN Y CONCLUSIONES}

El registro de un nuevo sitio de arte rupestre en el sector de bosque cordillerano viene a aumentar la acotada evidencia arqueológica que se conoce para Norpatagonia andina occidental. Las características iconográficas y la ubicación del sitio sugieren similitudes con la evidencia conocida en ambos lados de los Andes, apuntando a códigos visuales compartidos entre ambos espacios y que supone cierta interacción entre grupos $y / 0$ movilidad en tiempos tardíos. Su ubicación en un sector de paso cordillerano, cuya aptitud podemos interpretar a partir de su uso en épocas históricas, y su similitud con Santa Bárbara 1 en el paso de Vuriloche, sugieren relaciones de este tipo de registros con sectores de tránsito entre ambas vertientes andinas. Estilísticamente, nos encontramos ante una relativa continuidad en lo observado para épocas tardías en el Manso inferior de la vertiente oriental, lo que podría apuntar a una filiación histórico cultural vinculada de alguna forma con las sociedades cazadoras-recolectoras de la pampa y en el Holoceno tardío (Fernández et al. 2013).

Desde la óptica de los sitios de transición pampa-cordillera en la vertiente oriental, se ha planteado que el registro rupestre en el bosque occidental podría corresponder a movimientos para el aprovisionamiento de recursos vegetales por parte de grupos de las llanuras patagónicas (Bellelli et al. 2008). Por otro lado, la evidencia de contactos y movilidades transcordilleranas entre grupos huilliches, mapuches, pehuenches y tehuelches es abundante en épocas históricas (e.g. Rosales, 1657; Menéndez, 1900; Cox, 2012), por lo cual suponer que se trataría de vías recurrentes de tránsito es otra hipótesis plausible. Lo cierto es que la evidencia en las sierras selváticas occidentales es aún escasa y poco trabajada como para aventurar mayores interpretaciones.

Por otro lado, consideramos que los motivos registrados en Paredón Luisa no se asemejan a las pinturas de Morro Vilcún (Fig. 2), pues estas últimas presentan una amplia diversidad de motivos, tales como rombos, círculos, chevrones y un posible 
positivo de mano; por otra parte, y como han mencionado los autores, no se adscribirían al Estilo Grecas (Labarca et al. 2016). Al mismo tiempo, en Morro Vilcún además de pinturas, se ha reconocido nueve grabados, técnica ausente en Paredón Luisa. En dicho sitio, ubicado en un contexto costero, los depósitos estratigráficos evidencian aprovisionamiento de recursos marinos, y aunque no hay claridad de la temporalidad de las representaciones rupestres, se ha asociado parte de la ocupación a contextos canoeros tardíos del seno de Reloncaví, como Piedra Azul y Huenquillahue 2 (Mena et al. 2011; Labarca et al. 2016). Morro Vilcún está localizado a $160 \mathrm{~km}$ de los sitios del camino de Cochamó, y constituye la evidencia de arte rupestre más próxima en dirección al sur. En ese sentido, es posible que durante el Holoceno tardío existiesen en la vertiente occidental modos de vida diferentes y contemporáneos, que estuvieran desarrollando tradiciones rupestres diversas (Gaete et al. 2004; Reyes et al. 2009, 2019; Rivas \& Ocampo, 2010).

\section{PROYECCIONES}

La identificación de sitios en general, y de arte rupestre en particular, en la vertiente occidental andina de Norpatagonia ha tenido un avance intermitente, pero que ha levantado interés en la última década. El registro de sitios como los del camino de Cochamó (Podestá et al. 2008), Vuriloche (Dillehay \& Ocampo, 2016) y Morro Vilcún (Mena et al. 2011), comienza a delinear un paisaje desde el cual pensar un desarrollo a futuro. En ese sentido, una tarea prioritaria corresponde a la intervención estratigráfica de los contextos registrados al occidente de la cordillera, algo que ha dado resultados bastante satisfactorios en Morro Vilcún (Labarca et al. 2016) y Santa Bárbara 1 (Dillehay \& Ocampo, 2016). Además, sería oportuno ampliar el registro del arte rupestre, incluyendo una mayor cantidad de variables espaciales y técnicas.

Por otro lado, la expedición al camino de Cochamó permitió obtener diversas comunicaciones de pobladores respecto de posibles sitios en los ríos Los Morros, Leones y Manso, entre los que se cuentan tanto ubicaciones de sitios a cielo abierto donde se han registrado materiales que indicarían desarrollos alfareros, como aleros con material lítico y posiblemente nuevos registros de arte rupestre. Ya anteriormente se ha resaltado el hallazgo de un soporte de molienda (Labarca, 2007) y de una clava o toqui de piedra (Bellelli et al. 2008). En ese sentido, el desarrollo de nuevas campañas de identificación de sitios es otra tarea pendiente. En este contexto, el arte rupestre representa una puerta de entrada al estudio y comprensión de las dinámicas de ocupación del pasado. Profundizar las investigaciones dirigidas a esta materialidad podría significar un gran aporte en el entendimiento de la zona y particularmente de la relación entre poblaciones que ocuparon ambas vertientes de Los Andes.

\section{AGRADECIMIENTOS}

A la Fundación Sendero de Chile por financiar y guiar el viaje en el camino de Cochamó. A Osvaldo "Manchaca" Delgado, Alberto Orrego, Leticia Contreras y Lucas Ochsenius por guiarnos y acompañarnos en las labores de terreno. A Luisa Valderas y familia por llevarnos al sitio, compartir historias y recibirnos en su hogar. A los evaluadores anónimos cuyos comentarios ayudaron a mejorar este trabajo. Cualquier error u omisión es de exclusiva responsabilidad nuestra.

\section{BIBLIOGRAFÍA}

Acevedo, A., \& Franco, N. (2012). Aplicación de DStretch a imágenes digitales del arte rupestre de Patagonia (Argentina). Comechingonia Virtual, VI(2), 152-175.

Albornoz, A., \& Cúneo, E. (2000). Análisis comparativo de sitios con pictografías en ambientes lacustres boscosos de Patagonia Septentrional: Lagos Lácar y Nahuel Huapi (provincias de Neuquén y Río Negro). En M. M. Podestá \& M. De Hoyos (Eds.), Arte en las rocas: Arte rupestre, menhires y piedras de colores en Argentina (pp. 163-174). Buenos Aires: Sociedad Argentina de Antropología.

Bellelli, C., Scheinsohn, V., \& Podestá, M. M. (2008). Arqueología de pasos cordilleranos: un caso de estudio en Patagonia norte durante el Holoceno tardío. Boletín del Museo Chileno de Arte Precolombino, 13(2), 3755.

Cox, G. (1863) [2012]. Viaje en las regiones septentrionales de la Patagonia 1862 - 1863. Santiago: Cámara 
Chilena de la Construcción.

Dillehay, T. D., \& Ocampo, C. (2016). El Paso "Vuriloche" Chile-Argentina. Ruta de los Jesuitas. Puerto Montt: Universidad Austral de Chile.

Fernández, P., Carballido, M., Bellelli, C., \& Podestá, M. M. (2013). Tiempo de cazadores. Cronología de las ocupaciones humanas en el valle del río Manso inferior (Río Negro). En A. Zangrando, R. Barberena, A. Gil, G. Neme, M. Giardina, L. Luna, C. Otaola, S. Paulides, L. Salgán \& A. Tívoli (Comps.), Tendencias teórico-metodológicas y casos de estudio en la arqueología de la Patagonia, (167175). San Rafael: Museo de Historia Natural de San Rafael.

Gaete, N., Navarro, X., Constantinescu, F., Mera, C., Selles, D., Solari, M. E., Vargas, M. L.,... \& Durán, L. (2004). Una mirada al modo de vida canoero del mar interior desde Piedra Azul. Chungará, Revista de Antropología Chilena, Vol. Especial, 333-346.

Gradin, C. (1988). Caracterización de las Tendencias Estilísticas del Arte Rupestre de la Patagonia (Provincias de Río Negro, Chubut y Santa Cruz, República Argentina). Contribuciones al Estudio del Arte Rupestre Sudamericano, 2, 54-67.

Harman, J. (2008) [2005]. Using Decorrelation Stretch to Enhance Rock Art Images. Recuperado de: http:// dstretch.com/AlgorithmDescription.html (acceso 1.06.2012).

Infante, S. (2013). Cruce de los Andes por el viejo paso de Cochamó. Guía de Senderismo Patrimonial. Fundación Sendero de Chile.

Labarca, R. (2007). Informe de prospección arqueológica proyecto vial Río Manso (comuna de Cochamó, Región de Los Lagos, Chile).

Labarca, R., Mena, F., Prieto, A., Dupradou, T., \& Silva, E. (2016). Investigaciones arqueológicas en torno a los primeros registros de arte rupestre en Morro Vilcún. Boletín del Museo Chileno de Arte Precolombino, 21(2), 65-80.

Mena, F., González, E., \& Labarca, R. (2011). Primeros registros de arte rupestre en el litoral de Patagonia Septentrional chilena. Magallania (Punta Arenas), 39(2), 303-307.

Menéndez, F. (1900). Viajes de Fray Francisco Menéndez a Nahuel Huapi. Valparaíso: Gillet.

Menghin, O. (1957). Estilos del Arte Rupestre de Patagonia. Acta Praehistorica, 1, 57-87. Centro de Estudios Prehistóricos, Buenos Aires.

Moreno, P. I. (2004). Millennial-scale climate variability in northwest Patagonia over the last 15000 yr. Journal of Quaternary Science, 19, 35-47.

Podestá, M. M., Bellelli, C., Labarca, R., Albornoz, A., Vasini, A., \& Tropea, E. (2008). Arte rupestre en pasos cordilleranos del bosque Andino Patagónico (El Manso, Región de Los Lagos y Provincia de Río Negro, ChileArgentina). Magallania, 36(2), 143-153.

Reyes, O., Méndez, C., Maldonado, A., Velásquez, H., Trejo, V., Cárdenas, M., \& Abarzúa, A. M. (2009). El uso del espacio de cazadores recolectores y paleoambiente Holoceno en el valle del río Cisnes, región de Aisén, Chile. Magallania, 37(2), 7-23.

Reyes, O., Tessone, A., San Román, M., \& Méndez, C. (2019). Dieta e isótopos estables de cazadores recolectores marinos en los canales occidentales de Patagonia, Chile. Latin American Antiquity, 30(3), 550-568.

Rivas, P., \& Ocampo, C. (2010). La adaptación humana al bosque en la isla de Chiloé. Estrategias adaptativas en el litoral septentrional de los canales patagónicos. Actas del XVII Congreso Nacional de Arqueología, Valdivia, Tomo 2, pp. 1449-1460.

Rosales, D. (1657[1878]). Historia General del Reyno de Chile Flandes Indiano, Tomo III. Valparaíso: Imprenta del Mercurio. 